\title{
Fractal Dimension Analysis of Higher-Order Mode Shapes for Damage Identification of Beam Structures
}

\author{
Runbo Bai, ${ }^{1,2}$ Maosen Cao, ${ }^{2,3}$ Zhongqing Su, ${ }^{4}$ \\ Wiesław Ostachowicz, ${ }^{3}$ and $\mathrm{Hao}_{\mathrm{Xu}}{ }^{4}$ \\ ${ }^{1}$ Department of Engineering Mechanics, College of Water-Conservancy and Civil Engineering, \\ Shandong Agricultural University, Taian 271000, China \\ 2 Department of Engineering Mechanics, Hohai University, Nanjing 210098, China \\ ${ }^{3}$ Institute of Fluid Flow Machinery, Polish Academy of Sciences, 80-952 Gdańsk, Poland \\ ${ }^{4}$ Department of Mechanical Engineering, The Hong Kong Polytechnic University, Hung Hom, \\ Kowloon, Hong Kong \\ Correspondence should be addressed to Maosen Cao, mmcao@imp.gda.pl
}

Received 23 April 2012; Accepted 27 June 2012

Academic Editor: Ivan Bartoli

Copyright (C) 2012 Runbo Bai et al. This is an open access article distributed under the Creative Commons Attribution License, which permits unrestricted use, distribution, and reproduction in any medium, provided the original work is properly cited.

Fractal dimension analysis is an emerging method for vibration-based structural damage identification. An unresolved problem in this method is its incapability of identifying damage by higher-order mode shapes. The natural inflexions of higher-order mode shapes may cause false peaks of high-magnitude estimates of fractal dimension, largely masking any signature of damage. In the situation of a scanning laser vibrometer (SLV) providing a chance to reliably acquire higher-order (around tenth-order) mode shapes, an improved fractal dimension method that is capable of treating higher-order mode shapes for damage detection is of important significance. This study proposes a sophisticated fractal dimension method with the aid of a specially designed affine transformation that is able to obviate natural inflexions of a higher-order mode shape while preserving its substantial damage information. The affine transformed mode shape facilitates the fractal dimension analysis to yield an effective damage feature: fractal dimension trajectory, in which an abruptly risking peak clearly characterizes the location and severity of the damage. This new fractal dimension method is demonstrated on multiple cracks identification in numerically simulated damage scenarios. The effectiveness of the method is experimentally validated by using a SLV to acquire higher-order mode shapes of a cracked cantilever beam.

\section{Introduction}

Structure health monitoring and damage detection using vibrational characteristics have been a research topic in the aerospace, mechanical, and civil fields for last decades $[1,2]$. In this area of research, various damage detection methods have been developed from modal 


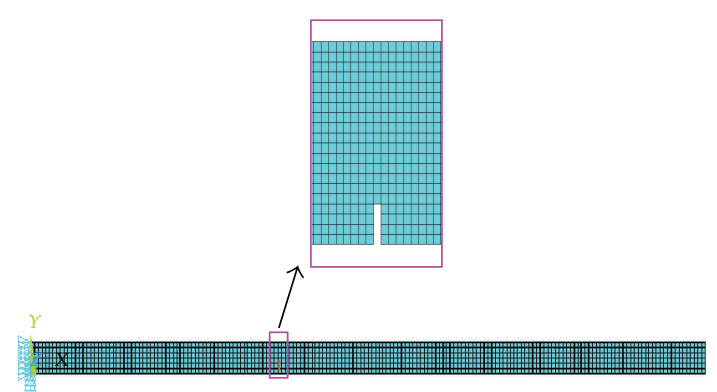

Figure 1: Numerical model of a cantilever beam with a single-edge crack described by $a=0.2$ and $\beta=0.4$ (Damage Scenario I).

parameters [3] such as natural frequency, mode shape [4], and mode damping. Among these parameters, it is commonly acknowledged that natural frequency has low sensitivity to small damage, mode damping is fairly difficult to acquire, and mode shape has the best potential to characterize damage [5]. Based on mode shape and its derivatives, a series of damage methods have been developed, most typically modal assurance criterion (MAC) [2], modal curvature and strain energy mode shape [6]. Nevertheless, some limitations in methods based on mode shapes have gradually been recognized; the most noteworthy of which is the lack of appropriate means to extract quantitative damage features from mode shapes [7]. In recent years, several new mathematical theories, for example, wavelet transform [8] and fractal dimension (FD) analysis [9] have been used to cope with mode shapes for identifying features of damage. In particular, FD analysis has attracted much attention in the field of structural damage detection.

FD analysis has become a burgeoning tool to provide insight into mode shapes for detecting damage [10-13]. The underlying principle of treating damage can be described as [10] follows: damage induces changes to the dynamic properties of a structure, consequentially causing irregularity of local mode shape; moreover, this irregularity can be characterized by an abrupt peak composed of high-magnitude estimates of FD, with the position and magnitude of the peak indicating the location and severity of the damage in a quantitative fashion. The efficacy of FD damage detection has been proved in many investigations. Hadjileontiadis et al. [10] utilized a moving window to successively cover the fundamental mode shape of a cantilever beam and calculate FD from the sampling points covered by the window, leading to a pointwise FD trajectory along the mode shape. In the trajectory, an abnormal peak composed of high-magnitude FD estimates reflects an irregularity of the local mode shape, indicating the location and severity of the damage. Li et al. [11] applied the FD to the first mode shape of simply supported steel beams with saw-cut cracks at different locations, and the results show the cracks were rightly identified. Shi et al. [12] implemented the FD analysis on the static deformation profile of one-crack and two-crack cantilever beamtype specimens and got satisfactory results of crack identification. The patulous application of the method to the fundamental two-dimensional (2D) mode shape of a simply supported cracked rectangular plate was investigated for damage identification by [5], where a 2D FD surface rather than a FD trajectory was available, and the peak in the surface predicted the location and quantification of the crack in the plate [14].

Most existing studies of FD damage detection are related to the fundamental mode shapes of beam-type structures due to their slight fluctuation in configuration and ease of measurement, but the application of the method to higher-order mode shapes is an interesting 


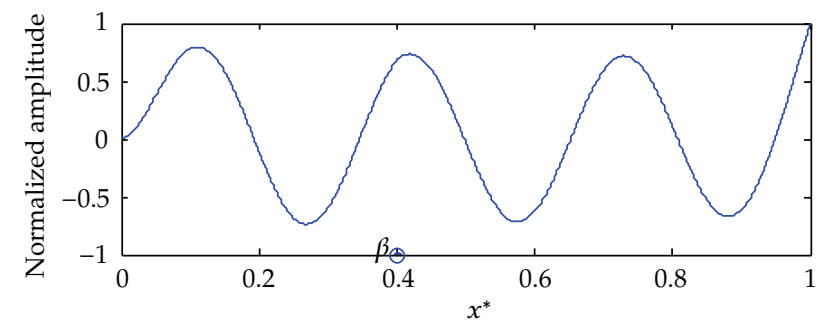

(a)

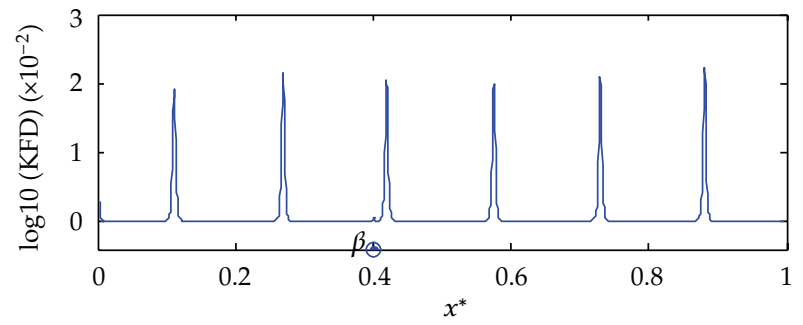

(b)

Figure 2: Seventh-order mode shape (a) and associated KFD trajectory (b) for Damage Scenario I (where $x^{*}$ denotes the normalized $x$-coordinate).

Table 1: Damage scenarios used in simulation.

\begin{tabular}{lcccc}
\hline Damage Scenario & I & II & III & IV \\
\hline Order of mode & 7th & 9 th & 11 th & 13th \\
Crack severity & $a=0.2$ & $a=0.2$ & $a=0.2$ & $a=0.2$ \\
Crack location & $\beta=0.4$ & $\beta=0.75$ & $\beta_{1}=0.3, \beta_{2}=0.7$ & $\beta_{1}=0.2, \beta_{2}=0.6, \beta_{3}=0.8$ \\
\hline
\end{tabular}

issue that is still unresolved well [15]. Several studies have addressed the limitations of FD in treating higher-order mode shapes to reveal damage $[16,17]$. The crucial point is that natural inflexions in a higher-order mode shape, as identified by the zero values of slope at those points, can create prominent false peaks in the FD trajectory [15], easily distorting the results of damage identification. Some studies have attempted to circumvent this problem that has been frustrating the use of FD in structural damage detection. Typically, Wang and Qiao [16] proposed a generalized fractal dimension method (GFD) with a flexible parameter to exaggerate the values of the $x$-coordinate of beam length, capable of detecting damage from the first three mode shapes of a cantilever beam. Qiao and Cao [17] proposed an approximate waveform capacity dimension (AWCD) and developed a regime of topological isomorphism to enable AWCD to reveal damage relying on the first three mode shapes of cantilever beams. Unfortunately, these methods have some imperfections in coping with higher-order mode shapes largely beyond the first three mode shapes. These higher-order mode shapes are hereinafter refered to as "around tenth-order" mode shapes. When encountering such a mode shape, these methods are usually inadequate to eliminate inflexion-induced false peaks in FD trajectory, and these false peaks are likely to mask any signature of damage.

Nowadays, it has become important to improve FD for damage detection on the basis of higher-order mode shapes. There are three reasons: (1) fractal theory has enormous potential to quantitatively characterize local irregularities or abnormities of a mode shape; 


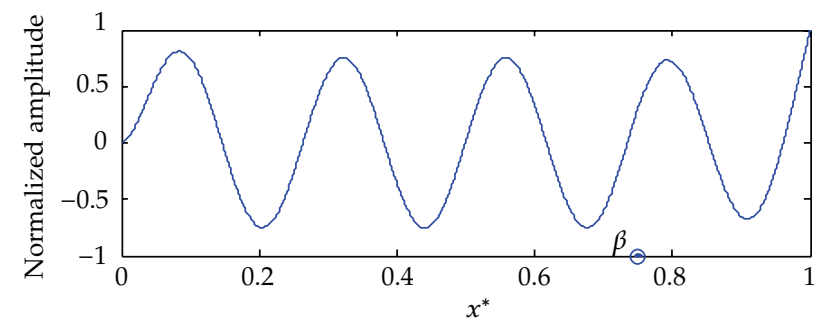

(a)

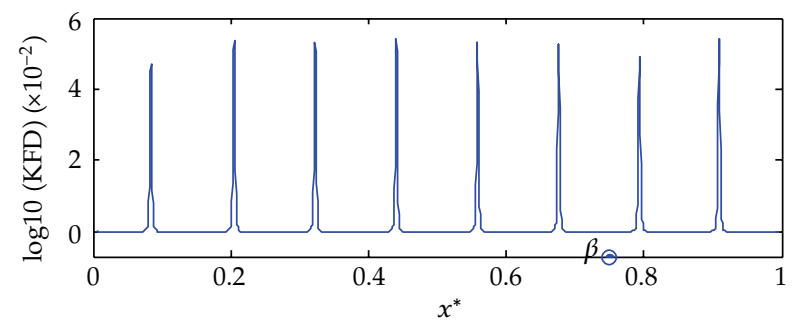

(b)

Figure 3: Ninth-order mode shape (a) and associated KFD trajectory (b) for Damage Scenario II (where $x^{*}$ denotes the normalized $x$-coordinate).

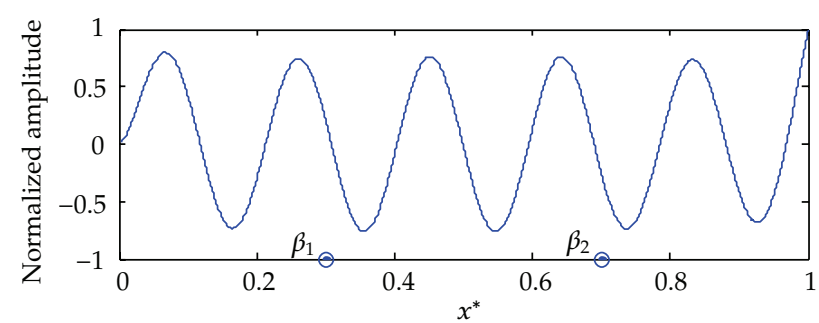

(a)

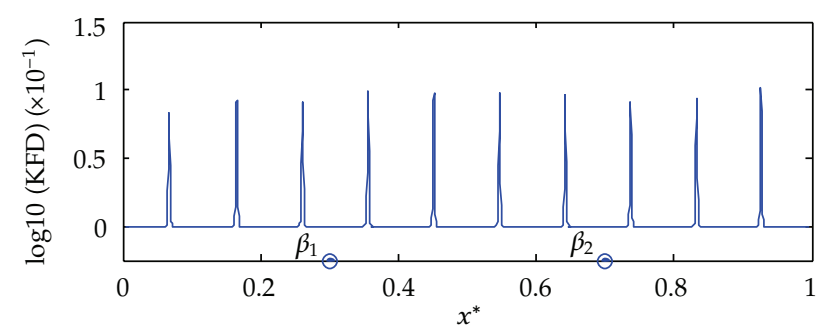

(b)

Figure 4: Eleventh-order mode shape (a) and associated KFD trajectory (b) for Damage Scenario III (where $x^{*}$ denotes the normalized $x$-coordinate).

(2) acquisition of around tenth-order mode shapes can be accurately achieved by modern experimental equipments, for example, scanning laser vibrometer (SLV); (3) higher-order mode shapes potentially convey richer damage information than lower-order ones. Given these conditions, this study aims to explore a sophisticated FD method that is capable of 


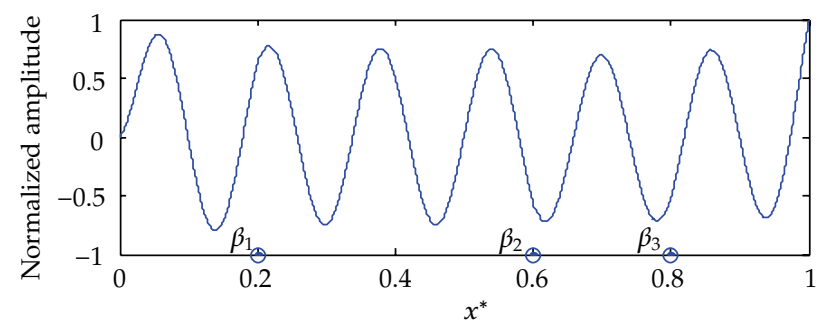

(a)

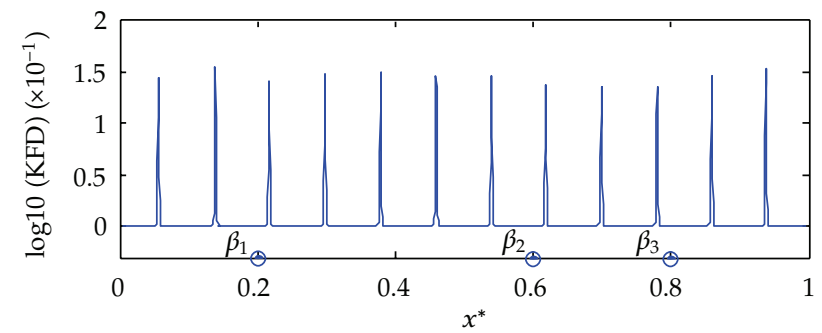

(b)

Figure 5: Thirteenth-order mode shape (a) and associated KFD trajectory (b) for Damage Scenario IV (where $x^{*}$ denotes the normalized $x$-coordinate).

tackling higher-order mode shapes for damage identification by overcoming the drawbacks of existing methods.

The organization of this paper is as follows. After this introduction, Section 2 examines the major deficiencies in existing FD methods for dealing with higher-order mode shapes to reveal damage. Section 3 presents a specially elaborated affine transformation that can convert a higher-order mode shape to a renascent one by preserving the substantial topological properties while eliminating the inflexions of the original mode shape. Section 4 provides a new FD method based on affine transform that is capable of characterizing damage using higher-order mode shapes. The proof-of-concept validation of the proposed method is given in Section 5, following which its effectiveness in damage identification in actual structures is experimentally investigated by using SLV to acquire the higher-order mode shapes.

\section{Fundamentals}

\subsection{Fractal Dimension}

The fractal dimension can be seen as a measure of the complexity of signals [18]. As a particular fractal dimension, the waveform fractal dimension is appropriate for characterizing the complexity of two-dimensional waveform signals [9]. Among the various waveform fractal dimensions available [18-22], the Katz's fractal dimension (KFD) [9] is probably the most commonly used due to its simplicity of concept and facilitation in computer implementations. Without loss of generality, the KFD is adopted in this study, and the method developed is applicable to other waveform fractal dimensions, for example, AWCD in [17]. 


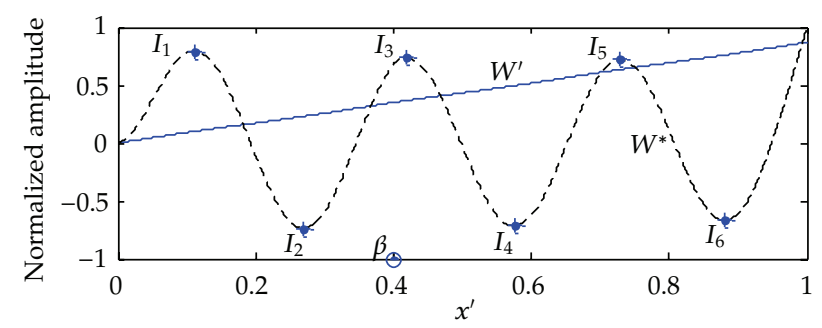

(a)

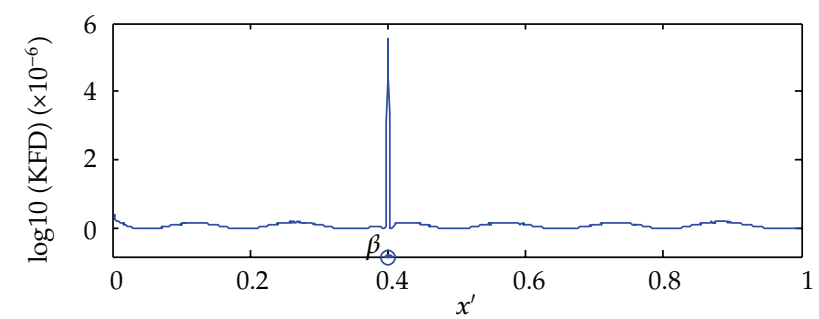

(b)

Figure 6: Effect of affine transformation on crack identification illustrated on the seventh-order mode shape for Damage Scenario I. (a) Affine transformed mode shape $\mathbf{W}^{\prime}$ and its original counterpart $\mathbf{W}^{*}$; (b) KFD trajectory showing a singular peak attributed to the crack.

The KFD of a two-dimensional waveform signal is defined by [9]

$$
\mathrm{KFD}=\frac{\log _{10}(n)}{\log _{10}(n)+\log _{10}(d / L)}
$$

where $n$ denotes the number of increments between adjacent sampling points of the signal under investigation, $d=\max$ dist $(1, i)$, the maximum distance between the beginning point, and the $i$ th point of the sampling sequence under investigation and $L$ is the sum of distances between successive points.

Although the KFD has been successfully applied to lower-order mode shapes for damage identification, its application to higher-order mode shapes is still somewhat problematic. To facilitate description of the problems, a numerical model of a cantilever beam with various crack scenarios is given in the following.

\subsection{A Cracked Beam Model}

A beam, $1 \mathrm{~m}$ long $(L), 0.02 \mathrm{~m}$ wide $(B)$, and $0.02 \mathrm{~m}$ thick $(H)$, is considered. The material data used are Young's modulus $E=70 \mathrm{GPa}$ and density $\rho=2700 \mathrm{~kg} / \mathrm{m}^{3}$. The numerical beam samples are built using 4-node 2D structural solid elements (PLANE42) in the commercial software ANSYS. The real constants are set by KEYOPT (3) $=3$ to specify the width of the elements. The numerical model of beams consists of two-thousand finite elements. The crack is described by two parameters: relative depth ratio (RDR), $a=h_{c} / H$, and relative location ratio (RLR), $\beta=L_{c} / L$, with $h_{c}$ and $L_{c}$ being the crack depth and crack location away from 


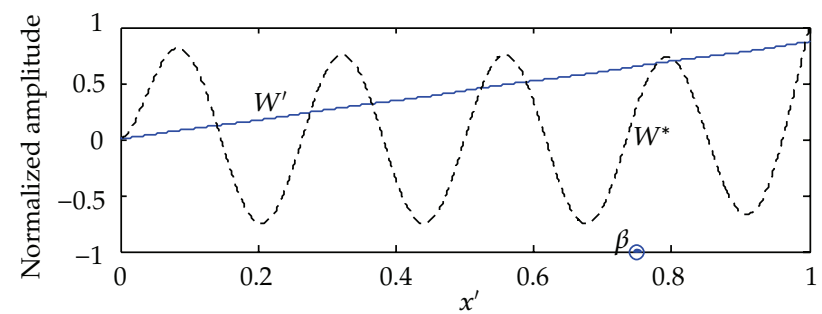

(a)

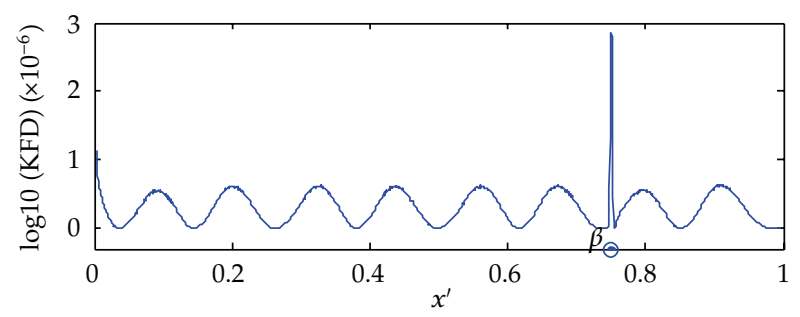

(b)

Figure 7: Crack identification on the ninth-order mode shape for Damage Scenario II. (a) Affine transformed mode shape $\mathbf{W}^{\prime}$ and its original counterpart $\mathbf{W}^{*}$; (b) KFD trajectory showing a singular peak attributed to the crack.

the clamped end, respectively. Each crack is modeled by reducing the thickness of the crosssection of a tiny segment of the beam, and various crack scenarios elaborated by adjusting parameters RDR and/or RLR are listed in Table 1. Figure 1 illustrates the finite element mesh of the cracked beam for Damage Scenario I described by $a=0.2$ and $\beta=0.4$, for which the manipulation of modal analysis can be adopted to generate higher-order mode shapes.

\subsection{Damage Feature: FD Trajectory}

A mode shape of a beam can be viewed as a particular two-dimensional waveform signal. The general procedure for applying the FD to a mode shape for damage detection is summarized as follows. A window with a fixed size, commonly containing a few sampling points, is utilized to cover the mode shape, and from the sampling points covered, an estimate of FD is evaluated and assigned at the midpoint of the window. This estimate quantitatively indicates the complexity of the window-covered segment of the mode shape. As the window slides point by point from the left to the right-hand end of a mode shape, an FD trajectory made up of a sequential of estimates appears. This FD trajectory represents a profile of the pointwise complexities of the mode shape. Damage causes increased irregularity or complexity of a local mode shape, manifested by high-magnitude estimates of FD, so a peak arising abruptly in the FD trajectory can predict the location and quantification of the damage. Thus, the FD trajectory can serve as a damage feature indicating the location and severity of the damage.

In this study, according to combined effects of sampling density and noise intensity, the sliding window is set to satisfy the following condition: covering 12 sampling points for a numerical mode shape and containing 10 sampling points for an experimental mode shape. 


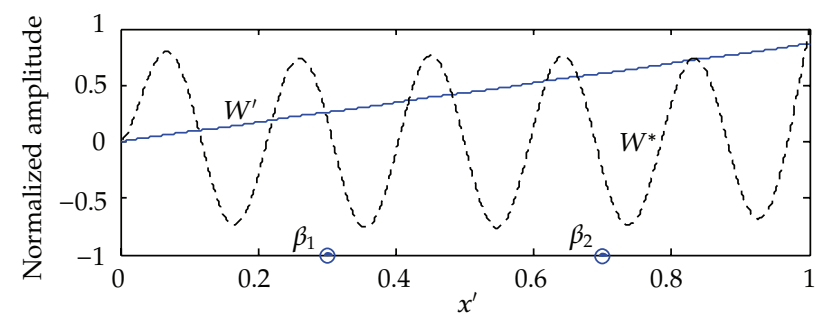

(a)

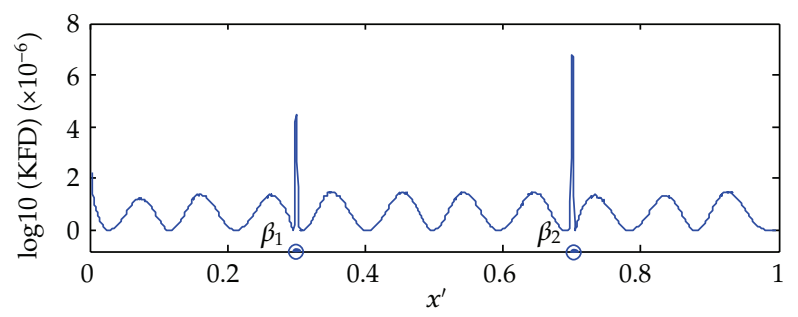

(b)

Figure 8: Crack identification on the eleventh-order mode shape for Damage Scenario III. (a) Affine transformed mode shape $\mathbf{W}^{\prime}$ and its original counterpart $\mathbf{W}^{*}$; (b) KFD trajectory showing two singular peaks attributed to the cracks.

\subsection{Deficiencies of FD with Higher-Order Mode Shapes}

As aforementioned, most successful applications of FD, for example, KFD, GFD, and AWCD, in damage detection are related to lower-order mode shapes [10-12], but the use of FD with higher-order (around tenth-order) mode shapes poses a challenge for existing FD methods. The crucial problem is that the inflexions, as illustrated in Figure 2(a), of higher-order mode shapes can cause false peaks of high-magnitude FD estimates, regardless of damage, which masks the genuine peak attributed to damage. For instance, regarding the seventhorder mode shape of the cracked beam model for Damage Scenario I, the negative effect of inflexion-induced false peaks in the KFD trajectory on damage characterization is illustrated in Figure 2, where the logarithm of KFD to base 10 is used in the $y$-coordinate for clarity in presentation. Clearly, the false peaks inflexions almost overwhelm the real peak attributed to the damage. This example illustrates the common problem of applying FD to higher-order mode shapes for damage detection. To further address this point, similar results concerning ninth-, eleventh-, and thirteenth-order mode shapes associated to Damage Scenarios II, III, and IV are shown in Figures 3,4, and 5, respectively. This inapplicability of FD to higherorder mode shapes for depicting damage limits the effectiveness of FD analysis in the field of structural damage detection.

\section{Affine Transformation of Higher-Order Mode Shapes}

\subsection{Affine Transformation}

An affine transformation [23] is mathematically defined as a transformation that preserves substantial topological properties such as collinearity (i.e., points on a line prior to the 


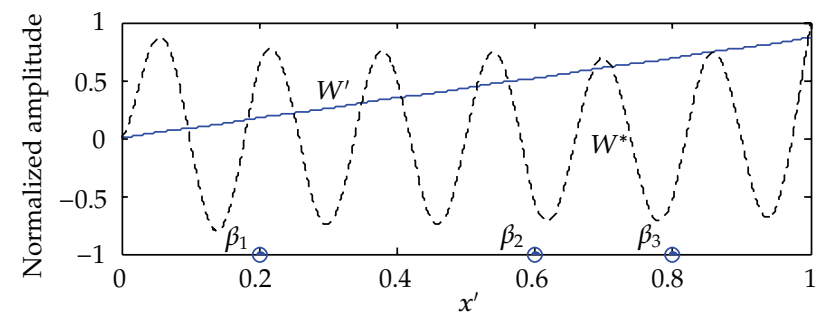

(a)

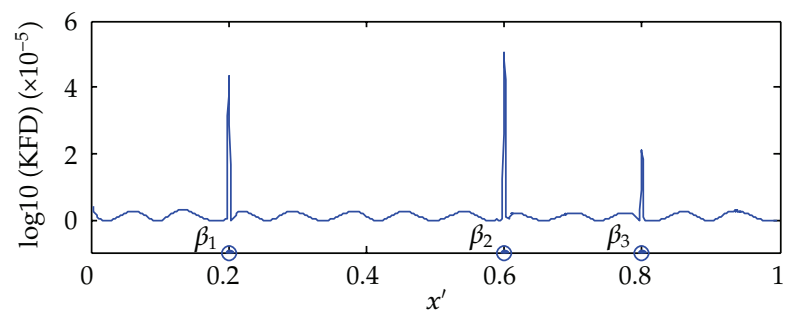

(b)

Figure 9: Crack identification on the thirteenth-order mode shape for Damage Scenario IV. (a) Affine transformed mode shape $\mathbf{W}^{\prime}$ and its original counterpart $\mathbf{W}^{*}$; (b) KFD trajectory showing three singular peaks attributed to the cracks.

transformation will lie on the line after the transformation), ratios of distances (i.e., proportions in lines are conserved by the transformation), and intersections of lines (i.e., an intersection of several lines will remain an intersection after the transformation despite probably changed angles between any pair of lines). Translation, reflection, expansion, shear, geometric contraction, dilation, rotation, similarity transformations, and spiral similarities are all affine transformations, as are their combinations [24].

For a two-dimensional point $(x, y)$ of an arbitrary waveform, its affine transformation can be carried out by means of premultiplying its homogeneous coordinates $(x, y, 1)$ by an affine transformation matrix $\mathbf{A}$, expressed as

$$
\left\{\begin{array}{l}
x^{\prime} \\
y^{\prime} \\
1
\end{array}\right\}=\mathbf{A}\left\{\begin{array}{l}
x \\
y \\
1
\end{array}\right\}, \quad \mathbf{A}=\left[\begin{array}{ccc}
c_{11} & c_{12} & c_{13} \\
c_{21} & c_{22} & c_{23} \\
0 & 0 & 1
\end{array}\right]
$$

In matrix $\mathbf{A}, c_{11}$ and $c_{22}$ are the scaling coefficients, $c_{12}$ and $c_{21}$ the shear coefficients, and $c_{13}$ and $c_{23}$ the translation coefficients. The affine transformation of a higher-order mode shape as a particular waveform gives rise to a renascent mode shape that preserves collinearity, ratios of distances and intersections but might alter the configuration of the original mode shape. Such properties of preservation and alteration of affine transformation are useful to retain damage content while obviating the inflexions of the original mode shape. 


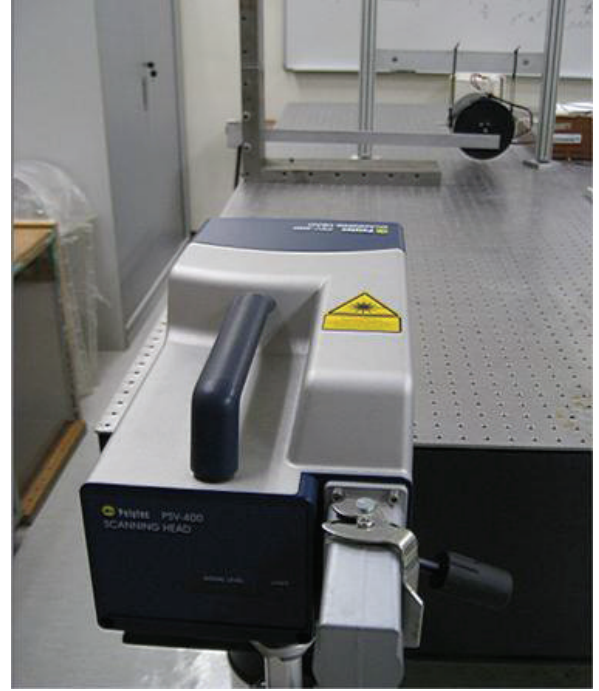

(a) Experimental setup

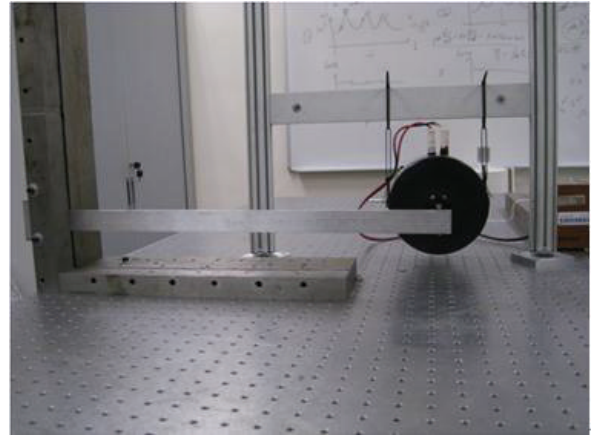

(b) Zoomed-in section showing excitation using an electromechanical shaker

Figure 10: Experimental measurement of mode shapes of cracked cantilever beam using SLV.

\subsection{Normalization of Higher-Order Mode Shapes}

In general, a higher-order mode shape is in the form of a sampling sequence, $\mathbf{W}=\left\{x_{i}, y_{i}\right\}_{i=1}^{n}$, where $x_{i}$ is the $i$ th sampling abscissa along the beam length, $y_{i}$ the amplitude of the mode shape at $x_{i}$, and $n$ the number of samplings. In physics, mode shape is a dimensionless quantity such that $\mathbf{W}^{c}=\left\{x_{i}, c y_{i}\right\}$, with $c$ being an arbitrary nonzero constant, has the same physical implication as $\mathbf{W}$; in contrast, in geometry $\mathbf{W}^{c}$ is a distinctive waveform from $\mathbf{W}$ such that it has different FD estimate from the latter, and hence there are probably nonunique FD characteristics for the same damage. It is necessary, therefore, to normalize a mode shape to reach a sole waveform, resulting in a unique FD characteristic to reflect damage. The method of normalization is expressed as

$$
x_{i}^{*}=\frac{x_{i}}{\max \left(\left\{x_{i}\right\}_{i=1}^{n}\right)}, \quad y_{i}^{*}=\frac{y_{i}}{\max \left(\left\{\left|y_{i}\right|\right\}_{i=1}^{n}\right)}
$$

where $x_{i}^{*}$ is the $i$ th sampling abscissa of the normalized mode shape and $y_{i}^{*}$ is the amplitude at $x_{i}^{*}$. After normalization, the original mode shape $\mathbf{W}$ yields a normalized mode shape $\mathbf{W}^{*}=$ $\left\{x_{i}^{*}, y_{i}^{*}\right\}_{i=1}^{n}$.

\subsection{Affine Transformation for Higher-Order Mode Shapes}

To obviate the inflexions of $\mathbf{W}^{*}=\left\{x_{i}^{*}, y_{i}^{*}\right\}_{i=1}^{n}$, a specific affine transformation matrix, $\mathbf{A}^{\prime}$, should be activated. The matrix $\mathbf{A}^{\prime}$ is built by using the elements $c_{11}=1, c_{21}=\sin \theta$, 


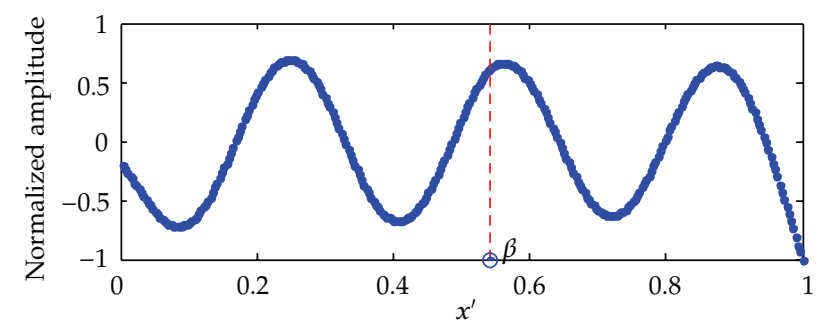

(a)

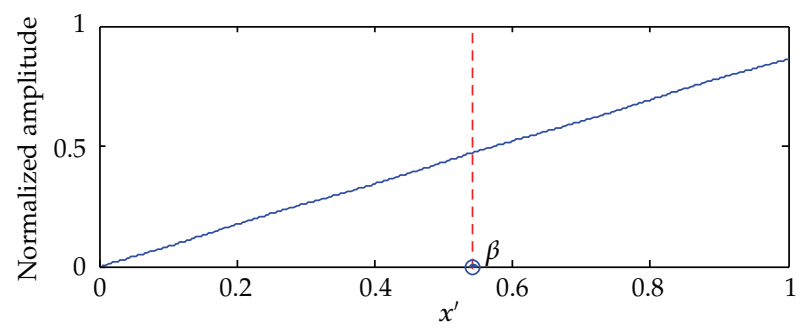

(b)

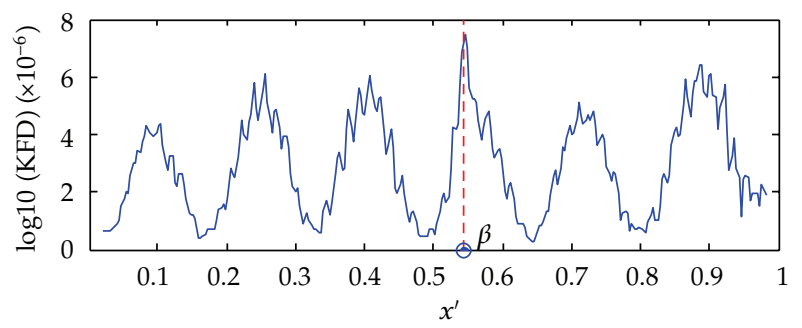

(c)

Figure 11: Experimental crack identification from SLV-measured seventh-order mode shape of cracked cantilever beam. (a) Normalized seventh-order mode shape; (b) affine transformed mode shape; (c) KFD trajectory showing a singular peak attributed to the crack.

$c_{22}=(\cos \theta) / k, c_{12}=c_{13}=c_{23}=0$ to specialize the ordinary affine transformation matrix, A, in (3.1), giving:

$$
\left\{\begin{array}{c}
x_{i}^{\prime} \\
y_{i}^{\prime} \\
1
\end{array}\right\}=\mathbf{A}^{\prime}\left\{\begin{array}{c}
x_{i}^{*} \\
y_{i}^{*} \\
1
\end{array}\right\}, \quad \mathbf{A}^{\prime}=\left[\begin{array}{ccc}
1 & 0 & 0 \\
\sin \theta & (\cos \theta) / k & 0 \\
0 & 0 & 1
\end{array}\right]
$$

In matrix $\mathbf{A}^{\prime},(\cos \theta) / k$ is the scaling coefficient for $y_{i}^{*}$, and $\sin \theta$ is the shear coefficient parallel to $y_{i}^{*} . k$ and $\theta$ are adjustment parameters for the scaling and shear coefficients, respectively. There are wide definition domains for $k$ and $\theta$ to satisfy the condition of obviating the inflexions of higher-order mode shapes. The flexibility in setting $k$ and $\theta$ is a distinctive feature of this affine transformation that makes it superior to other types of transformation. In this study, $k=100$ and $\theta=60^{\circ}$ are arbitrarily assigned to frame a specific affine transformation adopted for all higher-order mode shapes arising from both numerical and experimental damage cases. Based on this affine transformation, the normalized mode shape $\mathbf{W}^{*}$ 


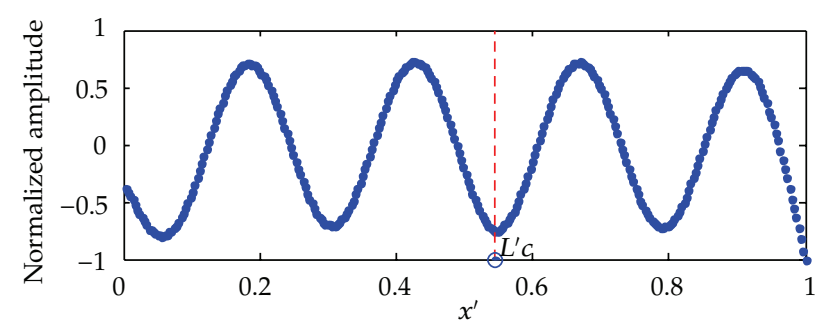

(a)

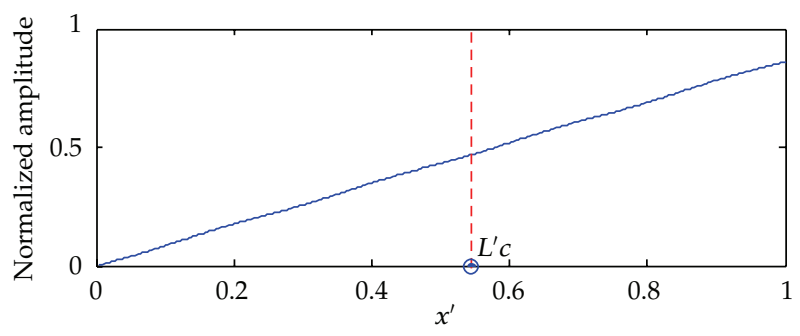

(b)

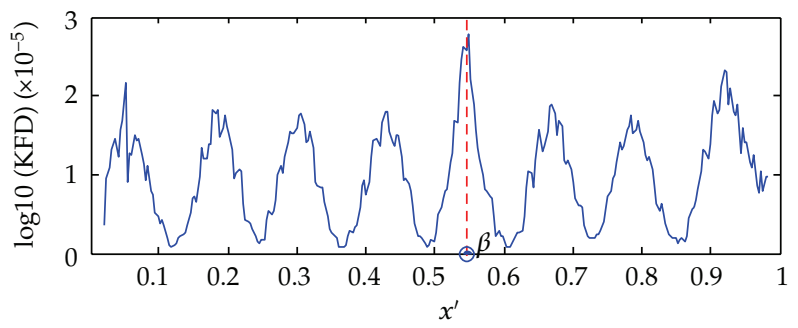

(c)

Figure 12: Experimental crack identification from SLV-measured ninth-order mode shape of cracked cantilever beam. (a) Normalized ninth-order mode shape; (b) affine transformed mode shape; (c) KFD trajectory showing a singular peak attributed to the crack.

creates a renascent mode shape $\mathbf{W}^{\prime}=\left\{x_{i}^{\prime}, y_{i}^{\prime}\right\}_{i=1}^{n}$ that preserves the damage information but eliminates its inflexions in $\mathbf{W}^{*}$.

By way of illustration, the proposed affine transformation is applied to the seventhorder mode shape shown in Figure 2(a), and the result is presented in Figure 6(a). In the figure, the normalized mode shape, $\mathbf{W}^{*}$, indicated by a dotted line, gives rise to an affine transformed mode shape, $\mathbf{W}^{\prime}$, designated by a solid line. It can be clearly observed that the six inflexions in $\mathbf{W}^{*}$ from $\mathbf{I}_{1}$ to $\mathbf{I}_{6}$ are unavailable in $\mathbf{W}^{\prime}$, and thus $\mathbf{W}^{\prime}$ can potentially serve as a reasonable alternative to $\mathbf{W}^{*}$ for use in damage identification. After the KFD analysis is carried out on $\mathbf{W}^{\prime}$, the resulting KFD trajectory shown in Figure 6(b) is produced. In the trajectory, a prominent peak, without interference from false peaks arising from inflexions, clearly indicates the location and severity of the crack. When the KFD trajectory in Figure 6(a) is compared to that from the original mode shape (Figure 2(b)), it can be concluded that the affine transformation significantly improves the performance of the FD methods for analyzing mode shapes for damage identification. 


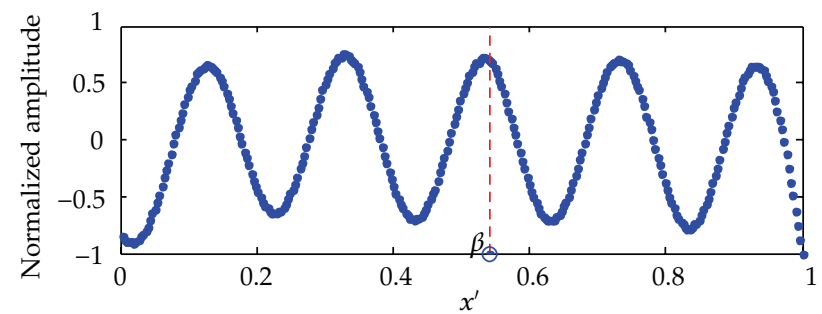

(a)

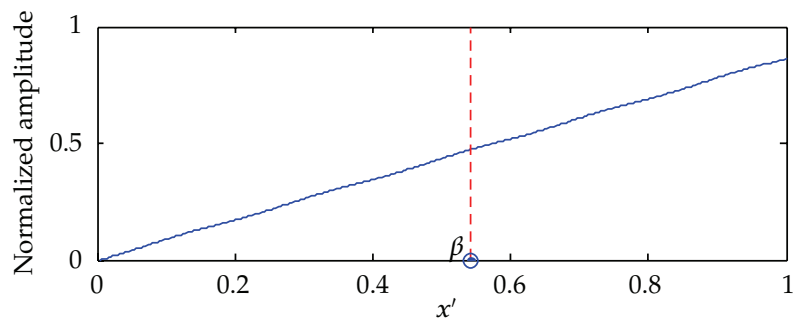

(b)

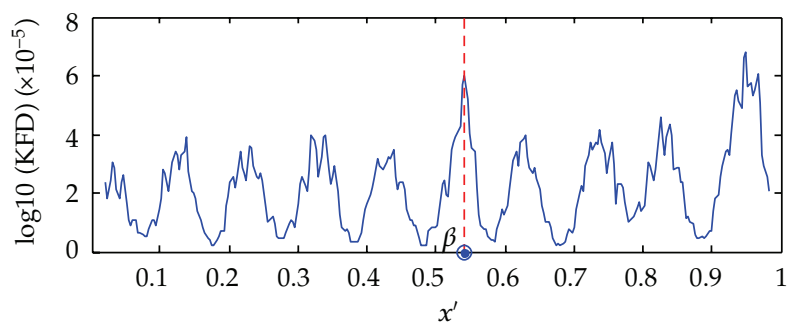

(c)

Figure 13: Experimental crack identification from SLV-measured eleventh-order mode shape of cracked cantilever beam. (a) Normalized eleventh-order mode shape; (b) affine transformed mode shape; (c) KFD trajectory showing a singular peak attributed to the crack.

\section{Concept-of-Proof Validation}

The previous analysis implies that the affine transformation-based FD analysis is a sophisticated method for detecting damage in beam-type structures. This method consists of three basic components: normalization, affine transformation, and FD analysis. The normalization described in (3.2) first runs on a higher-order mode shape acquired numerically or experimentally, giving rise to a normalized mode shape. Then the affine transformation given in (3.3) is employed to convert the normalized mode shape into a renascent mode shape, and finally the FD analysis is carried out on the renascent mode shape to yield an FD trajectory acting as a damage feature. The method is highlighted by the function of affine transformation to produce a new mode shape free of inflexions, offering a decent platform for the FD analysis to effectively reveal damage.

As a concept-of-proof validation, the proposed method is applied to Damage Scenarios II, III, and IV in Table 1, and the results are presented in Figures 7, 8, and 9, respectively. In Figures 7(a)-9(a), the normalized mode shapes and affine transformed mode shapes are marked by dotted and solid lines, respectively. In each figure, it is evident that the affine 
transformation obviates the inflexions of higher-order mode shapes dramatically. After the KFD analysis on the affine transformed mode shapes, the KFD trajectories are obtained, as shown in Figures $7(b)-9(b)$, respectively. In each figure, the predominant peaks, free of interference, clearly indicate the location and quantification of the cracks. When Figures $2-5$ are compared to Figures 6-9 for all Damage Scenarios listed in Table 1. It is concluded that the proposed method has strong capability to identify damage using higher-order mode shapes.

\section{Experimental Investigations}

The affine transformation-based FD analysis method for damage identification is experimentally investigated using a scanning laser vibrometer (SLV) to acquire higher-order mode shapes. A cantilever beam (aluminum 6061) of length $(L) 543 \mathrm{~mm}$, width $(B) 30 \mathrm{~mm}$, and height $(H) 8 \mathrm{~mm}$ is considered, as shown in Figure 10. A through-width crack, $1.2 \mathrm{~mm}$ long (along beam span) and $2 \mathrm{~mm}$ deep $(a=25 \%)$, located $293 \mathrm{~mm}(\beta=54 \%)$ away from the clamped end, is introduced into the beam. An out-of-plane monofrequency excitation at certain higher-order modal frequency, along $z$-direction at a point $525 \mathrm{~mm}$ from the clamped end, is applied by an electromechanical shaker (B\&K 4809). The selection of excitation frequency is based on a preliminary frequency response function (FRF) analysis. In the beam, out-of-plane velocities at all measurement points along the central line of the beam (evenly distributed with a spacing interval of $2.3 \mathrm{~mm}$ ) are captured from the intact surface of the beam, opposite to the surface where the damage is located, using an SLV (Polytec PSV-400). The experimental setup is photographed in Figure 10. The captured velocities are integrated to achieve the displacement responses of the beam using a FAST-SCAN function of the SLV.

To present a comprehensive insight into the capability of actual damage detection for the proposed method, the original experimental data free of any preprocessing, for example, denoising, are considered. The seventh-, ninth-, and eleventh- order mode shapes acquired by the SLV, after normalization, are presented in Figures 11(a), 12(a), and 13(a), respectively, and the associated affine transformed mode shapes are shown in Figures 11(b), 12(b), and 13(b), respectively. From the affine transformed mode shapes, the KFD trajectories are presented in Figures 11(c), 12(c), and 13(c) respectively. It can be observed from each KFD trajectory that the prominent peak of high-magnitude KFD values accurately indicates the location and quantification of the crack in the beam.

\section{Conclusions}

Fractal dimension (FD) analysis of mode shapes for damage identification is a new and potential research area. Despite the many advantages addressed in existing studies, the limitation of its inability to extract damage features from higher-order mode shapes is fairly noticeable. This fundamental study introduces an affine transformation to improve the existing FD method for damage identification, leading to a more sophisticated method. This method features the dramatic action of affine transformation in eliminating the inflexions of higher-order mode shapes, underpinning the FD analysis to competently reveal damage. The concept of proof of the method is demonstrated by numerical simulations; its effectiveness is further validated in experimental cases of cracked beams using a scanning laser vibrometer (SLV) to acquire higher-order mode shapes. The robustness against noise of the proposed method, the optimal selection of systematic parameters, and expansion to plate-type structures will be further investigated in future studies. 


\section{Acknowledgments}

M. Cao and W. Ostachowicz are grateful to a Marie Curie Industry Academia Partnership and Pathways Grant (Grant no. 251309 STA-DY-WI-CO) within the 7th European community Framework Programme. R. Bai wishes to acknowledge the National Natural Science Foundation of China for a research grant (Grant no. 50978084).

\section{References}

[1] F. K. Chang, "Introduction to health monitoring: context, problems, solutions, presentation," in Proceedings of the 1st European Pre-workshop on Structural Health Monitoring, Paris, France, 2002.

[2] Y. J. Yan, L. Cheng, Z. Y. Wu, and L. H. Yam, "Development in vibration-based structural damage detection technique," Mechanical Systems and Signal Processing, vol. 21, no. 5, pp. 2198-2211, 2007.

[3] C. R. Farrar and K. Worden, "An introduction to structural health monitoring," Philosophical Transactions of the Royal Society A, vol. 365, no. 1851, pp. 303-315, 2007.

[4] O. S. Salawu, "Detection of structural damage through changes in frequency: a review," Engineering Structures, vol. 19, no. 9, pp. 718-723, 1997.

[5] X. Jiang, Z. G. John Ma, and W. X. Ren, "Crack detection from the slope of the mode shape using complex continuous wavelet transform," Computer-Aided Civil and Infrastructure Engineering, vol. 27, no. 3, pp. 187-201, 2012.

[6] M. Cao and P. Qiao, "Novel Laplacian scheme and multiresolution modal curvatures for structural damage identification," Mechanical Systems and Signal Processing, vol. 23, no. 4, pp. 1223-1242, 2009.

[7] E. Sazonov and P. Klinkhachorn, "Optimal spatial sampling interval for damage detection by curvature or strain energy mode shapes," Journal of Sound and Vibration, vol. 285, no. 4-5, pp. 783$801,2005$.

[8] M. Cao and P. Qiao, "Integrated wavelet transform and its application to vibration mode shapes for the damage detection of beam-type structures," Smart Materials and Structures, vol. 17, no. 5, Article ID 055014, pp. 2337-2453, 2008.

[9] M. J. Katz, "Fractals and the analysis of waveforms," Computers in Biology and Medicine, vol. 18, no. 3, pp. 145-156, 1988.

[10] L. J. Hadjileontiadis, E. Douka, and A. Trochidis, "Fractal dimension analysis for crack identification in beam structures," Mechanical Systems and Signal Processing, vol. 19, no. 3, pp. 659-674, 2005.

[11] H. Li, Y. Huang, J. Ou, and Y. Bao, "Fractal dimension-based damage detection method for beams with a uniform cross-section," Computer-Aided Civil and Infrastructure Engineering, vol. 26, no. 3, pp. 190-206, 2011.

[12] J. Shi, X. Xu, J. Wang, and G. Li, "Beam damage detection using computer vision technology," Nondestructive Testing and Evaluation, vol. 25, no. 3, pp. 189-204, 2010.

[13] Y. H. An and J. P. Ou, "Experimental and numerical studies on damage localization of simply supported beams based on curvature difference probability method of waveform fractal dimension," Journal of Intelligent Material Systems and Structures, vol. 23, no. 4, pp. 415-4426, 2012.

[14] L. J. Hadjileontiadis and E. Douka, "Crack detection in plates using fractal dimension," Engineering Structures, vol. 29, no. 7, pp. 1612-1625, 2007.

[15] A. Katunin, "Fractal dimension-based crack identification technique of composite beams for on-line SHM systems," Machine Dynamics Research, vol. 34, no. 2, pp. 60-69, 2010.

[16] J. Wang and P. Qiao, "Improved damage detection for beam-type structures using a uniform load surface," Structural Health Monitoring, vol. 6, no. 2, pp. 99-110, 2007.

[17] P. Qiao and M. Cao, "Waveform fractal dimension for mode shape-based damage identification of beam-type structures," International Journal of Solids and Structures, vol. 45, no. 22-23, pp. 5946-5961, 2008.

[18] R. Esteller, G. Vachtsevanos, J. Echauz, and B. Litt, “Comparison of fractal dimension algorithms using synthetic and experimental data," in Proceedings of the 1999 IEEE International Symposium on Circuits and Systems (ISCAS '99), Orlando, Fla, USA, June 1999.

[19] T. Higuchi, "Approach to an irregular time series on the basis of the fractal theory," Physica D, vol. 31, no. 2, pp. 277-283, 1988.

[20] B. B. Mandelbrot, The Fractal Geometry of Nature, vol. 13, W. H. Freeman, San Francisco, Calif, USA, 1982. 
[21] F. Normant and C. Tricot, "Method for evaluating the fractal dimension of curves using convex hulls," Physical ReviewA, vol. 43, no. 12, pp. 6518-6525, 1991.

[22] P. Paramanathan and R. Uthayakumar, "An algorithm for computing the fractal dimension of waveforms," Applied Mathematics and Computation, vol. 195, no. 2, pp. 598-603, 2008.

[23] G. Stols and H. Bernitz, "Reconstruction of deformed bite marks using affine transformations," Journal of Forensic Sciences, vol. 55, no. 3, pp. 784-787, 2010.

[24] R. W. Sharpe, Differential Geometry, vol. 166, Springer, New York, NY, USA, 1997. 


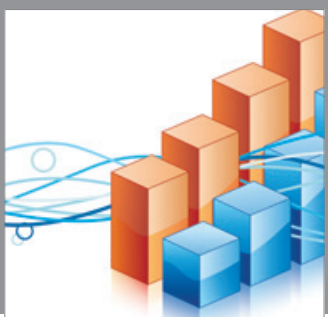

Advances in

Operations Research

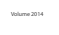

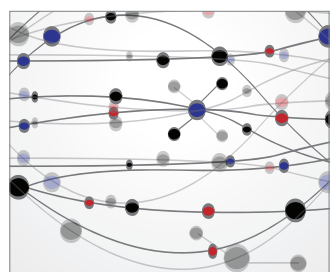

\section{The Scientific} World Journal
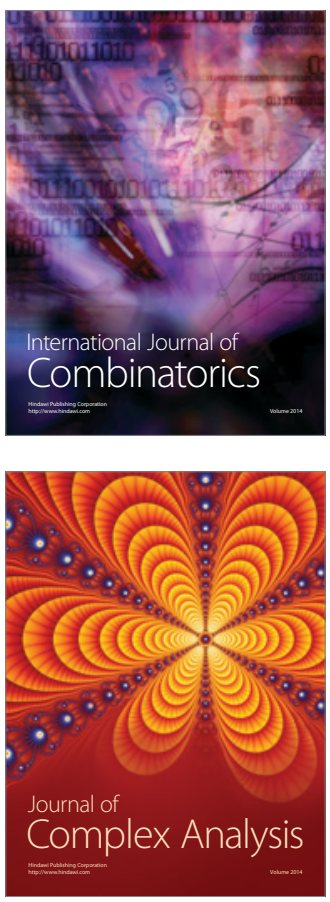

International Journal of

Mathematics and

Mathematical

Sciences
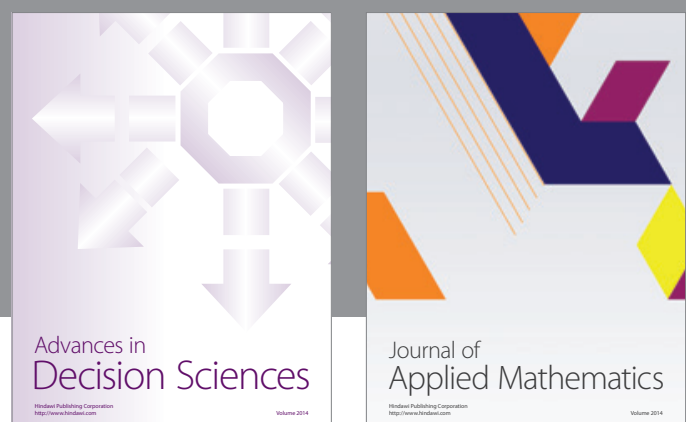

Journal of

Applied Mathematics
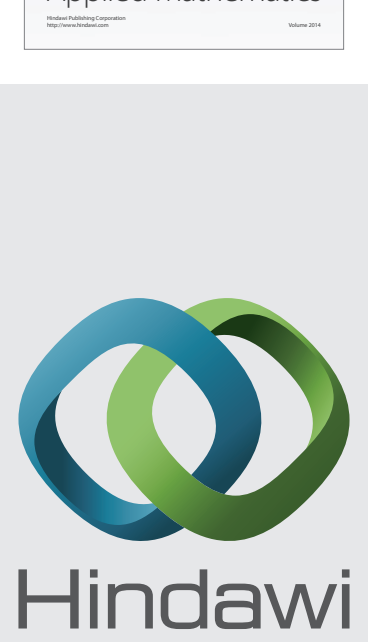

Submit your manuscripts at http://www.hindawi.com
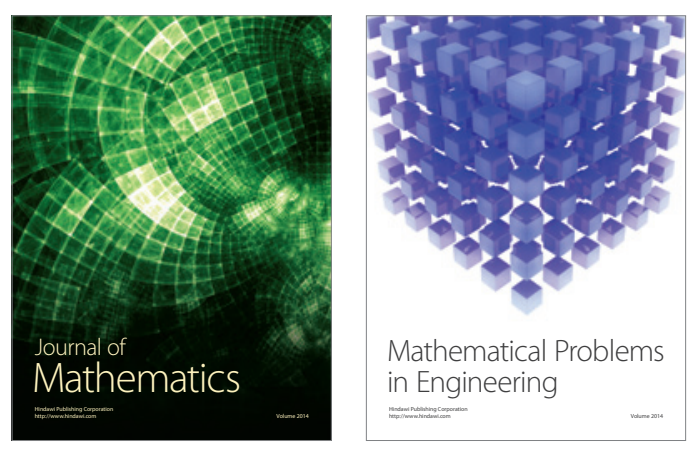

Mathematical Problems in Engineering
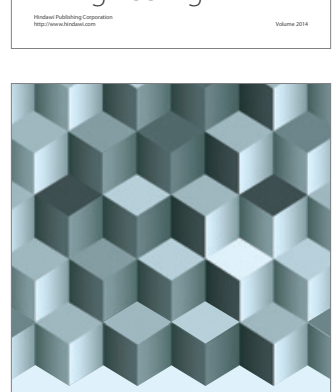

Journal of

Function Spaces
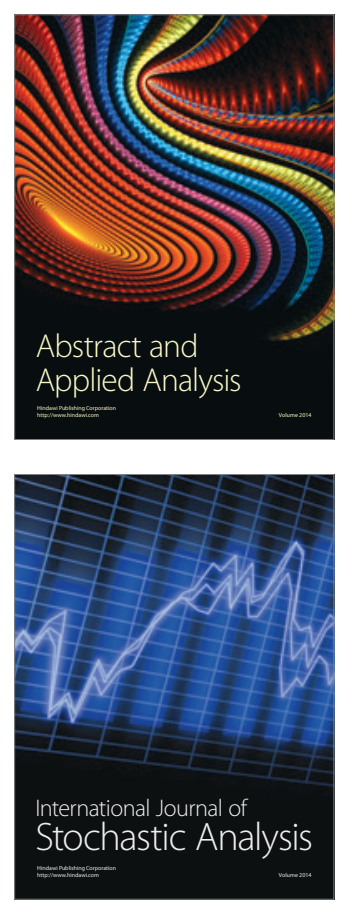

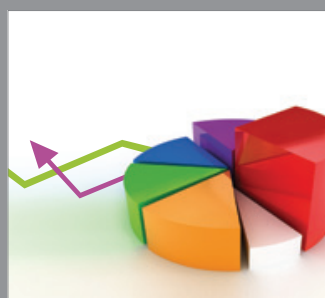

ournal of

Probability and Statistics

Promensencen
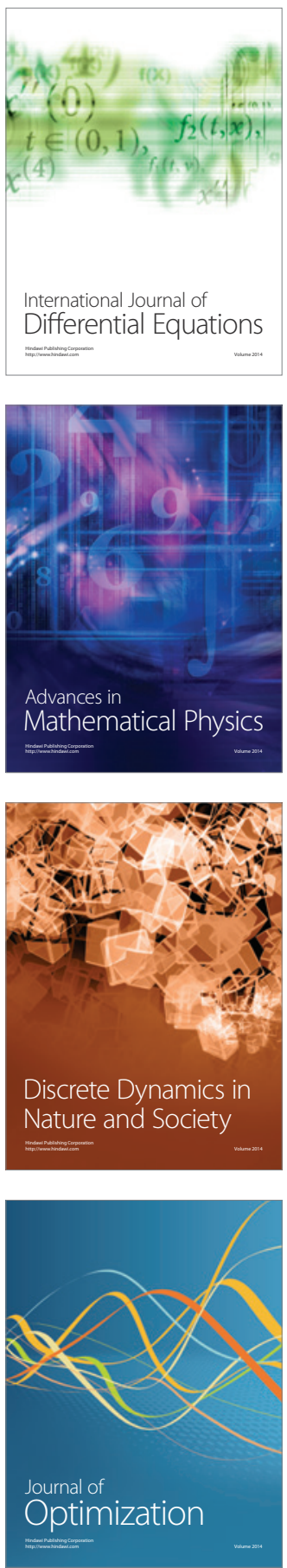